\title{
Faults Diagnosis of Self-validating Air Data Sensing System Based on Ensemble EMD and Multiclass RVM
}

\author{
Fu Changfeng ${ }^{1}$, Shen Zhengguang ${ }^{2}$, Han Lianfu ${ }^{1}$ and Liu Chao ${ }^{1}$ \\ ${ }^{1}$ Northeast Petroleum University / Daqing 163318, China \\ ${ }^{2}$ Beijing Institute of Automatic Control Equipment / Beijing 100076, China \\ E-Mails: shen_z_g@163.com, fuchangfeng8001@126.com
}

\begin{abstract}
Aiming at the non-linear fault features extraction, multiple fault patterns classification, and uncertain reasoning problem in faults diagnosis, a novel strategy by using multiclass relevance vector machine (RVM) coupled with ensemble empirical mode decomposition (EMD) for faults diagnosis of the self-validating air data sensing (SVADS) system is proposed. The working principle of the EEMD, especially the improved anti-mode mixing and the clear physical meaning of the decomposed intrinsic mode function (IMF), is emphasized for distinct faults features extraction, in which the energy and cutting ratio feature is picked up and different faults types can be validly distinguished from each other. The multiclass RVM is then employed for the faults diagnosis of multiple faults types in SVADS system, in which its advantages of multi-patterns simultaneous outputs, small sample learning and uncertain description of classification results by a form of probability are fully used. Based on the prototype design and failure mode analysis of the SVADS system, a real experimental system is designed, and then the fault-free and faulty data sample are collected to verify the performance of the proposed strategy. From the anti-mode mixing capacity of ensemble EMD and fault diagnosis accuracy of multiclass $R V M$ under small sample, the performance comparison among different methods or different sample number is done. Results demonstrate that the proposed scheme provides a better solution to the faults diagnosis of SVADS system.
\end{abstract}

Keywords: faults diagnosis; ensemble EMD; multiclass RVM; air data sensing system

\section{Introduction}

Recently National Aeronautics and Space Administration (NASA) has developed the flush air data sensing (FADS) system, which has replaced the traditional Pitot tube with distributed-mounted pressure points, in order to meet the needs of aircraft stealth capabilities, redundancy design, and high Mach number flight [1-2]. However, in realworld flight, the FADS system cannot acquire its own working status which will directly decide whether the current raw measurements value (RWV) can apply to the following flight control system, i.e., the on-line fault self-detection and self-diagnosis, data selfrecovery and status self-estimation functions are absent. Based on some previous work [36], a novel prototype of self-validating air data sensing (SVADS) systems is proposed to validate the output of the air data system and further to provide the reliability of the measurement value, in which it combines the strong points of both FADS technology and self-validating technology and its functional architecture is shown in Figure 1, and this paper will center on the fault diagnosis strategy.

Received (July 11, 2016), Review Result (October 5, 2016), Accepted (January 19, 2017) 


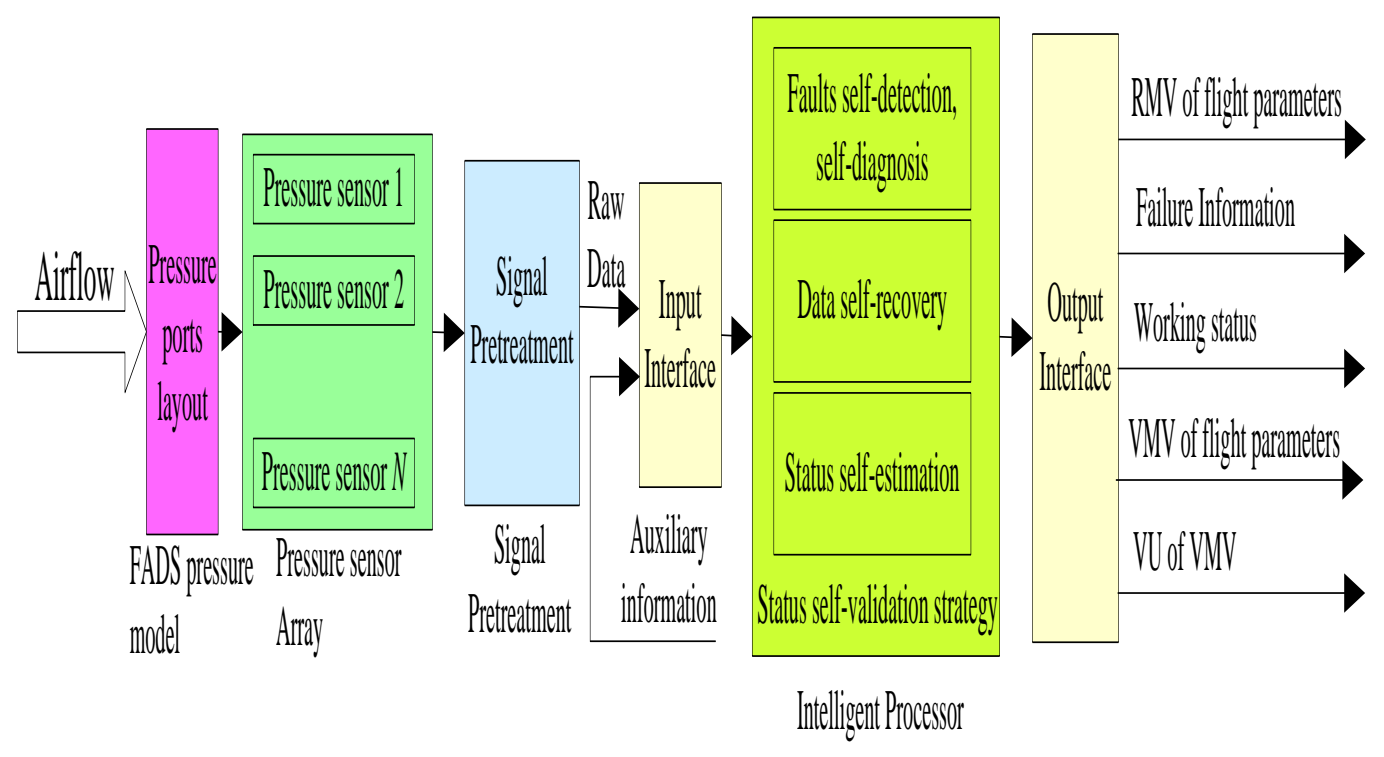

Figure 1. Construction Models of SVADS System

Once the computed flight parameters are invalid, it should give failure alarm and the key issue is how to judge measurement value unreliability with prompt solution, otherwise a serious flight disaster may be occurred when the wrong air data are used to the flying control system [7]. At this time, the incorrect measurements should be detected, identified and isolated to avoid its continuous propagation by using the previous faults self-detection methodology [8-9]. Further, some detailed faults information such as the type of faults is very needed, which can benefit the further device maintenance and can also be ready for latter data recovery under faults. Therefore, the faults diagnosis is the key part of SVADS system, in order to extract the faults features and implement faults patterns classification. However, the SVADS system has its own difficulties, and traditional methods cannot be directly applied. Firstly, the SVADS system concludes multiple pressure measuring channels, and each channel has complex faults modes such as the blockage of pressure ports, pipe pressure leak, faults of pressure sensors and faults of circuits; secondly, the faults feature of each mode is nonlinear, which the difficulty in faults feature extraction is bigger; thirdly, the mount of actual faults data is very little, and the small sample problem needs to be solved; lastly, the obtained fault information includes some uncertain extent and the classification results should be uncertain. Presently, some faults diagnosis algorithms have been proposed based on empirical mode decomposition (EMD) [10], neural networks [11], and relevance vector machine (RVM) [12]. Based on the adaptive decomposition of signals in frequency domain, EMD has applied to some feature exaction; however, its mode mixing problem is not suitable for nonlinear faults feature exaction of this paper. The neural networks can be used for the nonlinear problem, however, it needs a large amount of training samples data, and the uncertainty of classification results is absent. Although RVM can output some uncertainty of classification results, and process the small sample and non-linear problem, however, it only can output single fault pattern by establishing single model, and the implementation of multiple faults modes classification needs to be built multiple classifiers, which will increase the computational burden and affect the real-time performance of faults diagnosis. The essence of faults diagnosis is both the faults features extraction and the pattern classification, therefore, a better faults diagnosis model of SVADS system should be established for multiple faults patterns classification, good classification accuracy and little computational burdens under small sample and non-linear challenge, and reveal the uncertainty of classification results from the objective view of probability theory. 
Aiming at the above shortcomings of existing faults diagnosis methods, this paper proposes a sparse multiclass relevance vector machine (MCRVM) coupled with ensemble empirical mode decomposition (EMD) to implement the faults diagnosis of SVADS system, in which EEMD is very suitable to extract the non-linear faults features based on its adaptive signal decomposition and anti-mode mixing performance relative to the traditional EMD, and MCRVM is exactly employed for multiple classification under small sample situation. Compared with the traditional single model single classification of RVM, MCRVM can handle multiple faults patterns classification simultaneously with fine real-time performance by establishing only one model, and further it also provides the possibility of each classified fault mode in an objective way, and good classification performance under small sample problem. The proposed EEMD-MCRVM based faults diagnosis strategy is different from traditional pattern classification methods, and it is particularly suitable for uncertainty problem of faults diagnosis area. The SVADS system is relatively complicated due to multiple parameters and the complex correlation among them, and there are few achievements on this aspect. Our research was supported by the National Natural Science Foundation of China.

\section{Methodology}

\subsection{Faults Diagnosis Strategy Overview of SVADS System}

The faults diagnosis strategy of SVADS system mainly includes the features extraction of non-linear faults and design of faults patterns classifier, and the whole process consist of the training and testing phase of faults diagnosis model. Aiming at the simultaneous processing of multiple fault patterns and uncertain expression of the classification results, the EEMD-MCRVM based faults diagnosis model of SVADS system is proposed and the functional block diagram is shown in Figure 2.

In Figure 2, the overall work is generalized into the off-line establishment of faults diagnosis model and on-line test or validation. Firstly, the distributed pressure measurements of all channels are obtained based on the experimental platform setup of the SVADS system, in which the data sample under fault-free situation and faults are both pick up. Secondly, non-linear features of faults are extracted by using EEMD based faults features extraction algorithm, and the obtained feature can be used to describe the corresponding faults types of the SVADS system exactly and also be distinguished from each other. Detailed principle of non-linear faults features extraction by using EEMDbased model would be introduced in Section 2.2. Lastly, based on the extracted faults features sets which imply the relationship between faults features and their faults patterns, an optimized classifier can be designed by using the MCRVM based pattern classification theory, in order to implement the classification decision of different faults types. Detailed principle of pattern classification by using MCRVM-based model would be introduced in Section 2.3. 


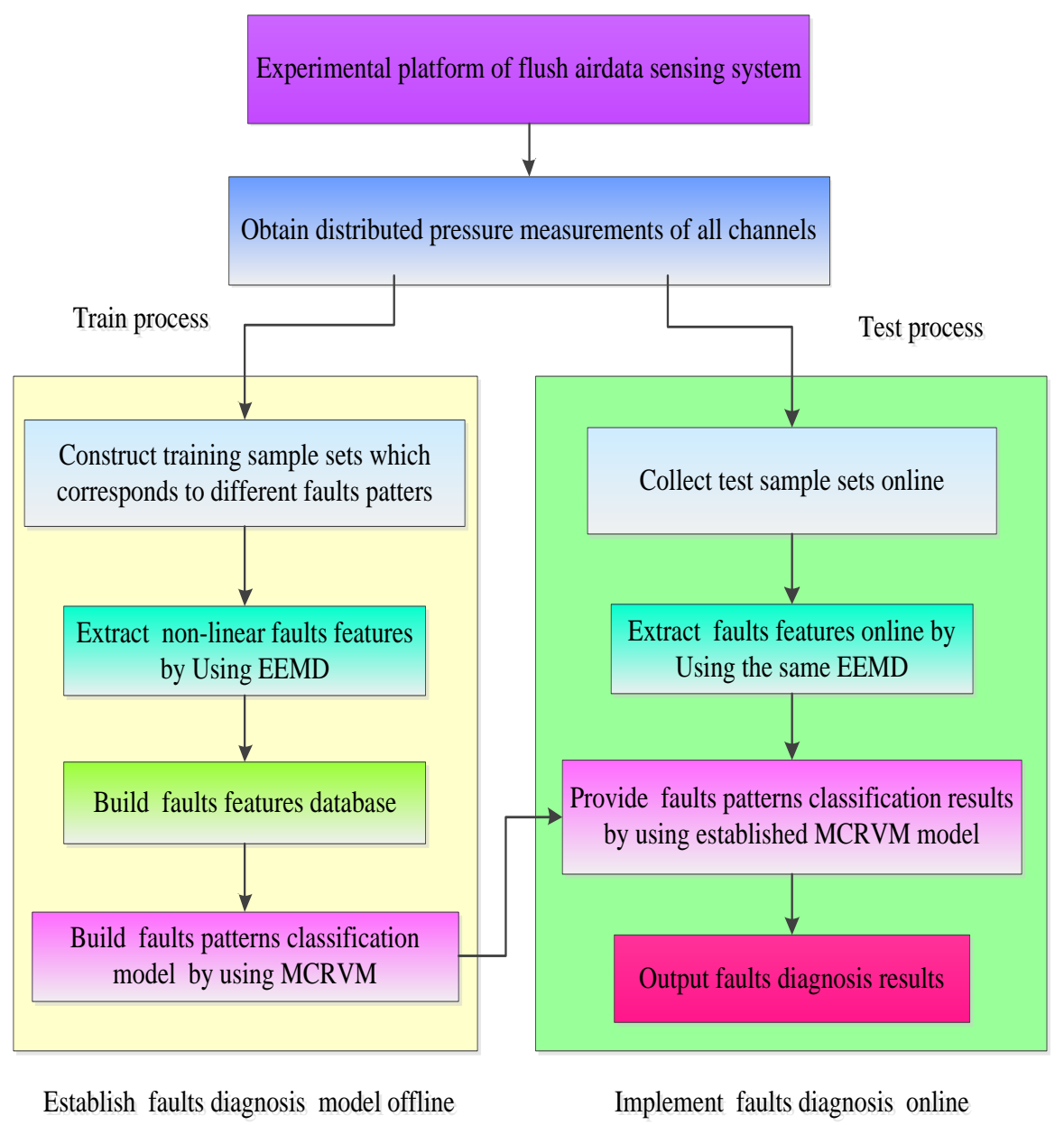

\section{Figure 2. Functional Block Diagram of Fault Diagnosis of SVADS System Using EEMD-MCRVM}

\subsection{Non-linear Fault Feature Extraction of SVADS System by Using EEMD}

The EEMD is used to divide an original signal into multiple components that is called as the intrinsic mode function (IMF) and residual in an adaptive decomposition way, in which each IMF is responded to the dynamic distinctive feature of original signal and the residual reflects the steady characteristic of original signal [13-14]. When certain pressure channel of the SVADS system is faulty, the measurements value series can produce some transient change which carries some special fault information, further, different fault type corresponds to different change of the above transient state. The advantages of the EEMD lie in the adaptive decomposition of signals and the decomposed IMFs can represent the non-linear faults with clear physical meanings, and it is very suitable for the non-linear fault feature extraction of the SVADS system.

\subsubsection{Mode Mixing and Basic Principle of EEMD}

Compared with the wavelet transform, the EMD is used for the adaptive multiresolution decomposition in some signal processing area. However, the EMD has the mode mixing problem which defined as a single IMF including oscillations of dramatically disparate scales, or a component of a similar scale residing in different IMFs. When the mode mixing occurs, it will make the physical meaning of individual IMF unclear, which implies that there may be different physical processes represented in a mode [15]. Aiming the problem of mode mixing in EMD, the improved EEMD based on 
the insight from the studies of the statistical properties of white noise was proposed [16], in which each trial consists of the traditional decomposition results of the signal plus a normally distributed white noise with a constant standard deviation and EMD behaves like a dyadic filter bank. Additionally, the above white noise could help data analysis in the EMD method. All these investigations promote the use of EEMD in feature extraction and analysis of complex signals.

The basic principle on which the EEMD algorithm is based is that the added normally distributed white noise will populate the whole time-frequency space uniformly in accordance with the constituting components of different scales. When a signal is added to this uniformly distributed white noise background, the components in different scales of the signal are automatically projected onto proper scales of reference established by the white noise in the background. In the EEMD algorithm, different white noise with mean zero and a constant standard deviation is added to the signal in each trial of decompositions and as a result, each of the noise-added decompositions consists of the signal and the added white noise. Thus, each individual trial will certainly produce very noisy results, but the noise in each separate trial is different. It can be decreased or even completely canceled out in the ensemble mean, given enough trials. The ensemble mean is treated as the true answer because only the signal persists as more and more trials are added to the ensemble. The EEMD principle advanced here is based on the following observations: 1) white noise is necessary to force the ensemble to find all possible solutions; the white noise makes the different scale signals reside in the corresponding IMFs, and render the resulting ensemble mean more meaningful. 2) a collection of white noise cancels each other out in an ensemble mean; therefore, only the signal can survive and persist in the final noise-added signal ensemble mean. 3) the decomposition with truly physical meaning is not the one without noise; it is designated to be the ensemble mean of a large number of trials consisting of the noise-added signal. More detailed description of EEMD can be found in [16].

Based on the above principle and observations, the EEMD algorithm is presented below and Figure. 3 is its flow chart.

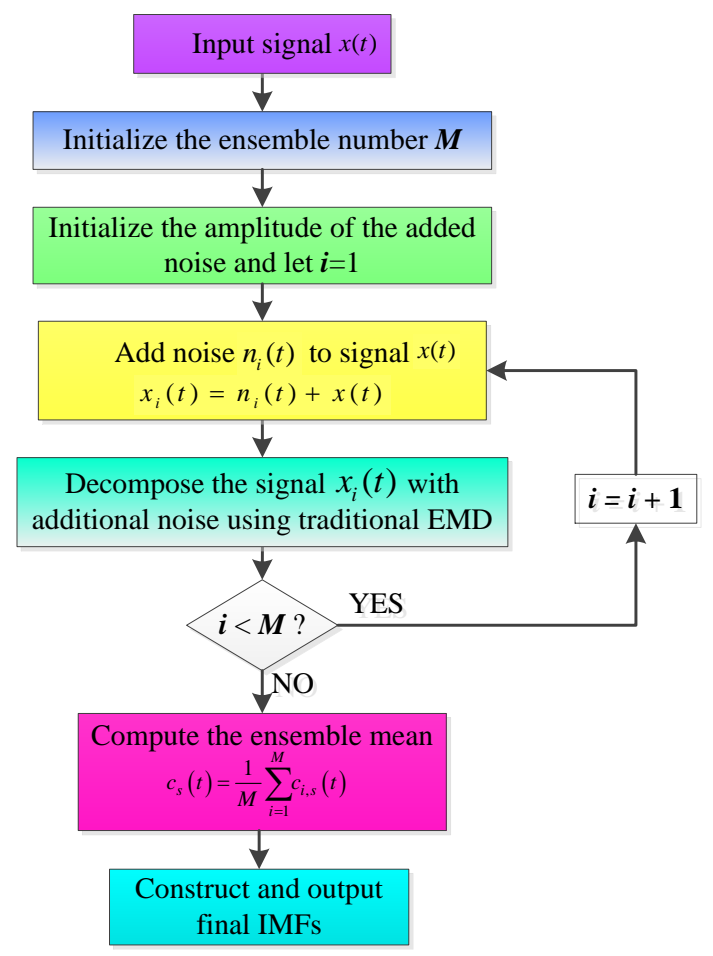

Figure 3. Flow Chart of EEMD Algorithm 
Step 1. Input the signal $x(t)$ and initialize the number of ensemble or trial steps $\boldsymbol{M}$;

Step 2. Initialize the amplitude parameter of the added white noise, meanwhile let $\boldsymbol{i}=1$ and start the EEMD algorithm;

Step 3. Obtain the signal by adding a white noise series with the given amplitude to the signal to be studied, and perform the $i$ th trial on the signal added white noise as shown in equation (1) wherein $n_{i}(t)$ indicates the $i$ th added white noise series, and $x_{i}(t)$ represents the noise-added signal of the $i$ th trial.

$$
x_{i}(t)=n_{i}(t)+x(t)
$$

Step 4. Decompose the noise-added signal $x_{i}(t)$ into $\boldsymbol{N}$ components of IMFs by using traditional EMD method, and these IMFs are noted as $c_{i, s}(t)$ as shown in equation (2) wherein $S$ is the number of decomposed IMFs, $r_{i, s}(t)$ is the residual, $c_{i, s}(t)$ is the $s$ th IMF of $i$ th trial process $(\mathrm{i}=1,2, \ldots, \mathrm{M}, \mathrm{s}=1,2, \ldots, S)$.

$$
x_{i}(t)=\sum_{s=1}^{S} c_{i, s}(t)+r_{i, s}(t)
$$

Step 5. Repeat $M$ times of the procedure (3) and (4), and IMFs set can be collected as shown in equation (3).

$$
\left[\left\{c_{1,1}(t), c_{1,2}(t), \cdots, c_{1, s}(t)\right\},\left\{c_{2,1}(t), c_{2,2}(t), \cdots, c_{2, s}(t)\right\}, \cdots,\left\{c_{M, 1}(t), c_{M, 2}(t), \cdots, c_{M, s}(t)\right\}\right]
$$

Step 6. Calculate the ensemble mean $c_{s}(t)$ of the $M$ trials for each IMF component using equation (4), and the mean is exactly taken as the final IMF of original signals.

$$
c_{s}(t)=\frac{1}{M} \sum_{i=1}^{M} c_{i, s}(t)
$$

Step 7. Construct and output final IMFs which are expressed as $c_{1}(t), c_{2}(t), \cdots, c_{S}(t)$.

The basic principle of EEMD and physical meaning of extracted IMF components has been introduced above; however, the ensemble number of trial steps and the amplitude of the added white noise are two parameters which are needed to be set. When small amplitude of the white noise is added, the final IMF has higher accuracy. But, if the amplitude is too small, the problem of mode mixing will not be controlled effectively. There are no specific equations reported in the literature to guide the choice of the amplitude, and it is suggested to be about $20 \%$ of the standard deviation of the input signal [16]. As for the parameter of ensemble number, the error of IMF components can be decreased by increasing the ensemble number; therefore, the ensemble number of a few hundred will lead to a fine result.

\subsubsection{Faults Features Extraction Based on Sensitive IMF Selection}

After performing EEMD on a signal, a series of IMFs are then obtained. A part of these IMFs are sensitive to faults of SVADS system, but some are not. Therefore, sensitive IMFs including fault-related information should be selected and irrelevant IMFs eliminated or improved to construct a more accurate faults features vector. Aiming at above difficulty, a method for faults features extraction based on EEMD is proposed, which is based on the difference of energy on certain IMFs components between the faults and fault-free signals of SVADS system. The procedure of feature extraction by using EEMD algorithm is presented below.

Step 1. Input the sample data of certain pressure channel which and the pressure measurement value series are noted as $\boldsymbol{X}=\left[\begin{array}{llll}x_{1} & x_{2} & \cdots & x_{N}\end{array}\right]$ wherein $N$ is length of sample. To ensure the final energy feature which indicates different faults types are not affected by signal amplitude, the normalization needs to be done by using equation (5), wherein $D_{\sigma}$ is the standard deviation and $E(\boldsymbol{X})$ is the mean value of test sample. 


$$
\tilde{\boldsymbol{X}}=D_{\sigma}^{-1}[\boldsymbol{X}-\boldsymbol{E}(\boldsymbol{X})]
$$

Step 2. Complete the signal decomposition of sample $\boldsymbol{X}$ by using EEMD and obtain seven IMFs called as $\mathrm{IMF}_{1}, \mathrm{IMF}_{2}, \ldots, \mathrm{IMF}_{7}$ and a residual called as $\mathrm{IMF}_{8}$.

Step 3. Enhance the signal quality of IMFs and residual, in order to make the feature of faults more clear. The enhanced value $\overline{\mathrm{I}} \overline{\mathrm{M}} \overline{\mathrm{F}}_{i, j}$ can be acquired by using equation (6) wherein $\mathrm{IMF}_{i, j}$ is the $j$ th original decomposition value of the $i$ th IMF component and $T h r_{\mathrm{IMF}_{i}}$ is the cutting threshold set by equation (7). The cut ratio cut $_{i}$ of IMFs and residual is represented as shown in equation (8).

$$
\begin{gathered}
\overline{\mathrm{I}} \overline{\mathrm{F}}_{i, j}= \begin{cases}\mathrm{IMF}_{i, j} & \left|\mathrm{IMF}_{i, j}\right| \geq T h r_{\mathrm{IMF}_{i}} \\
0 & \left|\mathrm{IMF}_{i, j}\right|<T h r_{\mathrm{IMF}_{i}}\end{cases} \\
\operatorname{Thr}_{\mathrm{IMF}_{i}}=\sqrt{\frac{1}{N} \sum_{j=1}^{N} \mathrm{IMF}_{i, j}^{2}} \\
\text { cut }_{i}=\frac{\operatorname{Num}\left(\overline{\mathrm{I}} \overline{\mathrm{F}}_{i, j} \neq \mathrm{IMF}_{i, j}\right)}{N}
\end{gathered}
$$

Step 4. Construct feature vectors of nonlinear faults. To extract the fault feature and implement the flowing pattern classification, the normalized energy feature and the average cutting ratio feature of IMF components and residual are computed by using equation (9) and (11). The final feature vectors of test sample are then constructed as

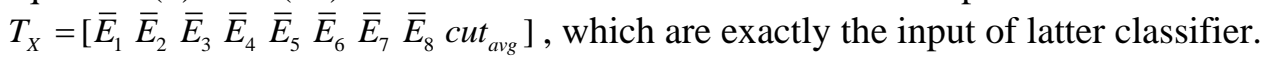

$$
\begin{gathered}
E_{i}=\int\left|\overline{\mathrm{I}} \overline{\mathrm{M}}_{i}\right|^{2} d t=\sum_{j=1}^{N}\left|\overline{\mathrm{I}} \overline{\mathrm{M}}_{i, j}\right|^{2} \\
\bar{E}_{i}=\frac{E_{i}}{\sqrt{\sum_{i=1}^{8}\left|E_{i}\right|^{2}}} \\
\text { cut }_{\text {avg }}=\frac{1}{8} \sum_{i=1}^{8} \text { cut }_{i}
\end{gathered}
$$

\subsection{Faults Patterns Classification of SVADS System by Using MCRVM}

Based on the previous extracted faults features vectors, the multiple faults pattern classifier can be established by using MCRVM, and different fault types can be diagnosed as different probabilistic output, and fault pattern with largest probability is final diagnosis result. The basic principle of the MCRVM and its detailed application in SVADS are follows.

\subsubsection{Multiple-class Problem and Basic Principle of MCRVM}

To identify the key elements of a data set, the relevance vector machines (RVM) model which is originally introduced by M. Tipping in order to employ a Bayesian treatment to produce probabilistic outputs for class membership or continuous target value estimation [17]. Although the RVM provides significantly competitive results in contrast to the traditional machine learning methods, its adaptation to the multi-class setting has been problematic and it can only process the binary class issue, due to the bad scaling of the type-II ML procedure with respect to the number of classes [18-19]. Aiming at the above constraint of a binary class problem and probabilistic outputs for class membership instead of the hard binary decisions given by the traditional classifier, the MCRVM is then proposed to expand the original RVM into the multiclass setting by introducing an auxiliary variable, which act as intermediate regression targets, and which naturally lead 
to the multinomial probit likelihood for the estimation of class membership probabilities. In this paper, the MCRVM algorithm is utilized to classify the faults after feature extraction. In the following, a brief introduction of MCRVM and indispensable equations are given.

Similar to the standard machine learning approach, in classification a training set $x=\left\{x_{i}, t_{i}\right\}_{i=1}^{N}$ should be given wherein $x \in R^{D}$ are the featured observations and $t \in\{1,2, \ldots, C\}$ are the respective class labels. More conveniently, our observations can be expressed as $\boldsymbol{x} \in R^{N \times D}$ from which we derive our training kernel $\boldsymbol{K} \in R^{N \times N}$ based on a dataset dependent kernel function. The training kernel captures our prior knowledge over the data sets and each row vector of $\boldsymbol{K}$ represents the similarity between current row sample and others. The learning process involves the inference of the model parameters $\boldsymbol{W} \in R^{N \times C}$, which by the quantity $\boldsymbol{W}^{T} \boldsymbol{K}$ act as a voting system to express which relationships of the data are important in order for our model to have appropriate discriminative properties [18]. Multiple class discrimination is achieved by the introduction of auxiliary variables $\boldsymbol{Y} \in R^{C \times N}$ that act as the regression targets of $\boldsymbol{W}^{T} \boldsymbol{K}$ following a standardized noise model [19] shown in equation (12).

$$
y_{c n} \mid \boldsymbol{w}_{c}, \boldsymbol{k}_{n} \sim N_{y_{c n}}\left(\boldsymbol{w}_{c}^{\mathrm{T}} \boldsymbol{k}_{n}, 1\right)
$$

When the regressors $\boldsymbol{W}$ express the weight with which a data point votes for certain specific class, the auxiliary variables $\boldsymbol{Y}$ express a class membership ranking system. The continuous nature of $\boldsymbol{Y}$ not only allows multiple class discrimination via the multinomial probit link $t_{n}=i$ if $y_{n i}>y_{n j} \forall j \neq i$, but also a probabilistic output for class membership via the resulting multinomial probit likelihood function shown in equation (13) wherein the $u \in N(0,1)$ and $\Phi$ are the Gaussian cumulative distribution function.

$$
P\left(t_{n}=i \mid \boldsymbol{W}, \boldsymbol{k}_{n}\right)=\varepsilon_{p(u)}\left\{\prod_{j \neq i} \Phi\left(u+\left(\boldsymbol{w}_{i}-\boldsymbol{w}_{j}\right)^{\mathrm{T}} \boldsymbol{k}_{n}\right)\right\}
$$

The diagram of the overall model is illustrated in Figure. 4, and this hierarchical Bayesian framework results in an implicit Student-t distribution which encourages sparsity by restricting the regression coefficients $\boldsymbol{W}$ posterior distribution around zero. The training procedure involves consecutive updates of the model parameters based on a standard Expectation Maximization (E-M) scheme. According to Figure 4, the regressors $\boldsymbol{W}$ closed form posterior can be derived shown in equation (14) wherein $\boldsymbol{A}_{c}$ is a diagonal matrix derived from the $c$ column of $\boldsymbol{A}$ which expresses the scales across samples. Thus given a class, the parameters across samples are updated based on the maximum a posteriori value shown in equation (15).

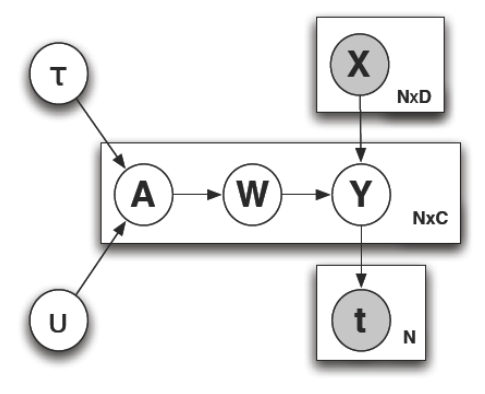

Figure 4. Plates Diagram of the Hierarchical Bayesian Model 


$$
\begin{aligned}
P(\boldsymbol{W} \mid \boldsymbol{Y}) & \propto P(\boldsymbol{Y} \mid \boldsymbol{W}) P(\boldsymbol{W} \mid \boldsymbol{A}) \\
& \propto \prod_{c=1}^{C} N\left(\left(\boldsymbol{K} \boldsymbol{K}^{\mathrm{T}}+\boldsymbol{A}_{c}\right)^{-1} \boldsymbol{K} \boldsymbol{y}_{c}{ }^{\mathrm{T}},\left(\boldsymbol{K} \boldsymbol{K}^{\mathrm{T}}+\boldsymbol{A}_{c}\right)^{-1}\right) \\
& \hat{\boldsymbol{w}}_{c}=\left(\boldsymbol{K} \boldsymbol{K}^{\mathrm{T}}+\boldsymbol{A}_{c}\right)^{-1} \boldsymbol{K} \boldsymbol{y}_{c}{ }^{\mathrm{T}}
\end{aligned}
$$

The posterior distribution of the auxiliary variables can be calculated by (15), which is a product of $C \times N$ dimensional conically truncated Gaussians. Here given a class $i$, the Estep $\forall c \neq i$ and $i$ th class are expressed as the equation (16) and (17) respectively wherein $\tilde{y}$ denotes the expected value.

$$
\begin{gathered}
\hat{y}_{c n} \leftarrow \hat{\boldsymbol{w}}_{C}{ }^{\mathrm{T}} \boldsymbol{k}_{n}-\frac{\varepsilon_{p(u)}\left\{N_{u}\left(\hat{\boldsymbol{w}}_{c}^{\mathrm{T}} \boldsymbol{k}_{n}-\hat{\boldsymbol{w}}_{i}^{\mathrm{T}} \boldsymbol{k}_{n}, 1\right) \Phi_{u}^{n, i, c}\right\}}{\varepsilon_{p(u)}\left\{\Phi\left(u+\hat{\boldsymbol{w}}_{i}^{\mathrm{T}} \boldsymbol{k}_{n}-\hat{\boldsymbol{w}}_{c}{ }^{\mathrm{T}} \boldsymbol{k}_{n}\right) \Phi_{u}^{n, i, c}\right\}} \\
\tilde{y}_{i n} \leftarrow \hat{\boldsymbol{w}}_{i}^{\mathrm{T}} \boldsymbol{k}_{n}-\left(\sum_{j \neq i} \tilde{y}_{j n}-\hat{\boldsymbol{w}}_{i}^{\mathrm{T}} \boldsymbol{k}_{n}\right)
\end{gathered}
$$

After the update of the hyperpriors $\tilde{\alpha}_{n c}$ which are again derived from the mean of a Gamma distribution, the closed-form posterior is shown in equation (18) and the mean of Gamma distribution is shown in equation (19).

$$
\begin{gathered}
p(\boldsymbol{A} \mid \boldsymbol{W}) \propto p(\boldsymbol{W} \mid \boldsymbol{A}) p(\boldsymbol{A} \mid a, b) \\
\propto p \prod_{c=1}^{C} \prod_{n=1}^{N} G\left(a+\frac{1}{2}, \frac{w_{n c}^{2}+2 b}{2}\right) \\
\tilde{\alpha}_{n c}=\frac{2 a+1}{w_{n c}^{2}+2 b}
\end{gathered}
$$

Each iteration of the learning procedure involves the updates from equation (19), (15), (16), (17) for each model parameter, until an appropriate convergence measure is satisfied. Detailed E-M scheme is similar to traditional RVM, which can be found in [19].

\subsubsection{Faults Diagnosis by Using MCRVM}

Based on above the basic principle analysis, MCRVM is in essence a Bayesian kernel learning theory under small samples, and its generalization ability with sparse model is fine. Therefore, the MCRVM is very suitable for the non-linear, small sample, multiclassification and uncertain problem in faults diagnosis of the SVADS system. The process of the faults diagnosis method based on MCRVM is described as follows:

Step 1. Build the standard database of faults features as training sample. Capture the pressure data of SVADS system under both different air data conditions and simulated damage types, and then implement the EEMD decomposition to construct feature vectors sets of nonlinear faults noted as $\boldsymbol{F}_{\text {train }} \in R^{m \times n}$ shown in equation (20) wherein $m$ is the number of data sample, and $n$ is the dimension number of faults features (here $n$ is equal to 9 which indicates the number of different energy features is 8 and the number of cutting ratio is 1$)$.

$$
\boldsymbol{F}_{\text {train }}=\left[\begin{array}{ccccccccc}
\bar{E}_{11} & \bar{E}_{12} & \bar{E}_{13} & \bar{E}_{14} & \bar{E}_{15} & \bar{E}_{16} & \bar{E}_{17} & \bar{E}_{18} & c u t_{\text {avg } 1} \\
\bar{E}_{21} & \bar{E}_{22} & \bar{E}_{23} & \bar{E}_{24} & \bar{E}_{25} & \bar{E}_{26} & \bar{E}_{27} & \bar{E}_{28} & c u t_{\text {avg } 2} \\
\bar{E}_{m 1} & \bar{E}_{m 2} & \bar{E}_{m 3} & \bar{E}_{m 4} & \bar{E}_{m 5} & \bar{E}_{m 6} & \bar{E}_{m 7} & \bar{E}_{m 8} & c u t_{\text {avgm }}
\end{array}\right]
$$

Step 2. Construct the classifier by using MCRVM. Taken the faults features database as the input of MCRVM, and the corresponding targeted faults patterns $\boldsymbol{T} \in \boldsymbol{R}^{k \times m}$ as output 
shown in equation (21), in which the failure type is consistent with sample data, the element in $\boldsymbol{T}$ is equal to 1 , otherwise 0 . The $k$ is number of faults types and its value is 5 which include the pressure fluctuation, jump, bias, constant output and fault-free situation. It is noted that the kernel parameter in MCRVM is optimized by 5-fold cross-validation.

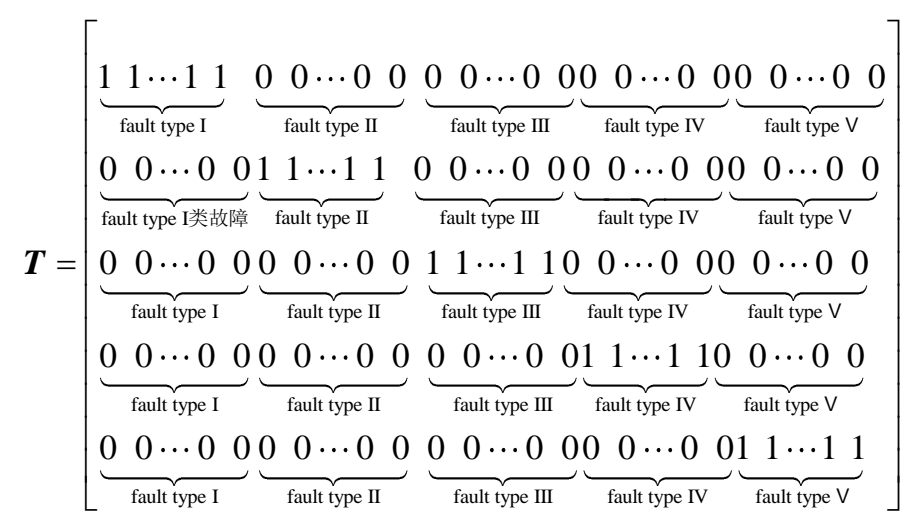

Step 3. Input the test sample into the established MCRVM based classifier. Similar to the previous feature extraction in Section 2.2, the original pressure data is processed as the test sample by using EEMD, and the corresponding feature vector is noted as $\boldsymbol{F}_{\text {test }} \in \boldsymbol{R}^{9}$.

Step 4. Output faults diagnosis results. Taken the $\boldsymbol{F}_{\text {test }}$ as the input of classifier, the probit likelihood estimation $\operatorname{Pro}_{i}(i=1,2, \ldots, k)$ which implies the current test sample belongs to different faults types can be obtained, and the fault pattern with maximum likelihood corresponds to the final faults diagnosis results exactly.

\section{Results and Discussion}

To verify the effectiveness of faults self- diagnosis strategy, the SVADS system experimental platform is designed and common faults coverage analysis is also stated. In this hardware platform, our proposed faults self- diagnosis method will be implemented.

\subsection{Experimental Setup and Basic Diagram}

Aiming at the cone-shape nose of the aerodynamic configuration based aircraft; a prototype of SVADS system has been preliminarily designed. The pressure ports layout employs eleven-point cross way, and the material thing of aircraft nose is shown in Figure 5. The prototype design of SVADS system can be described by the electric and airflow connection shown in Figure 6. The designed prototype of SVADS system are consist of airflow pipes that control the flow of air (dynamic pressure) to pressure sensor, eleven high-accuracy pressure sensors which use the vibration cylinder pressure transducer and output temperature-related analog and pressure-related frequency, the signal pickup and information processing unit which will be used to the measure pressure values and accomplish the self-validating algorithm, and 1553B based communication unit which can do data transmission to flying control system. 


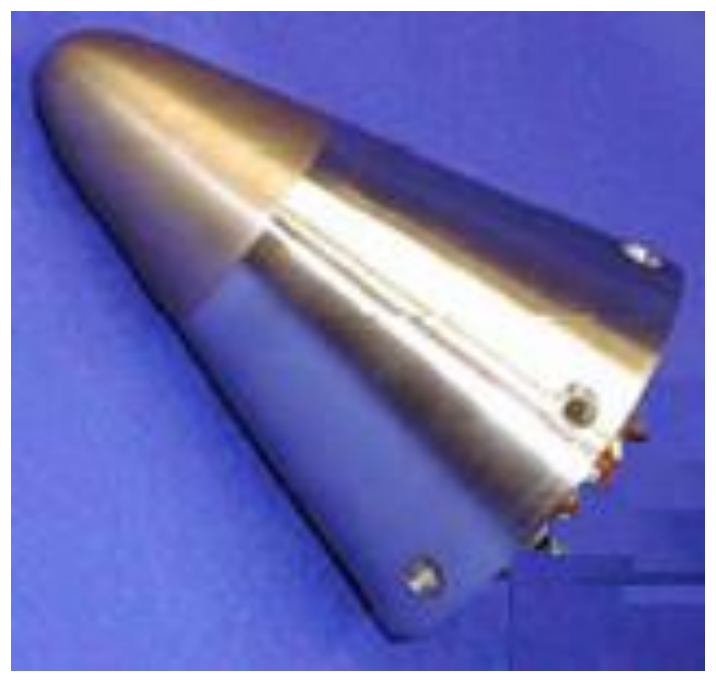

Figure 5. Material Thing Type of Aircraft Nose

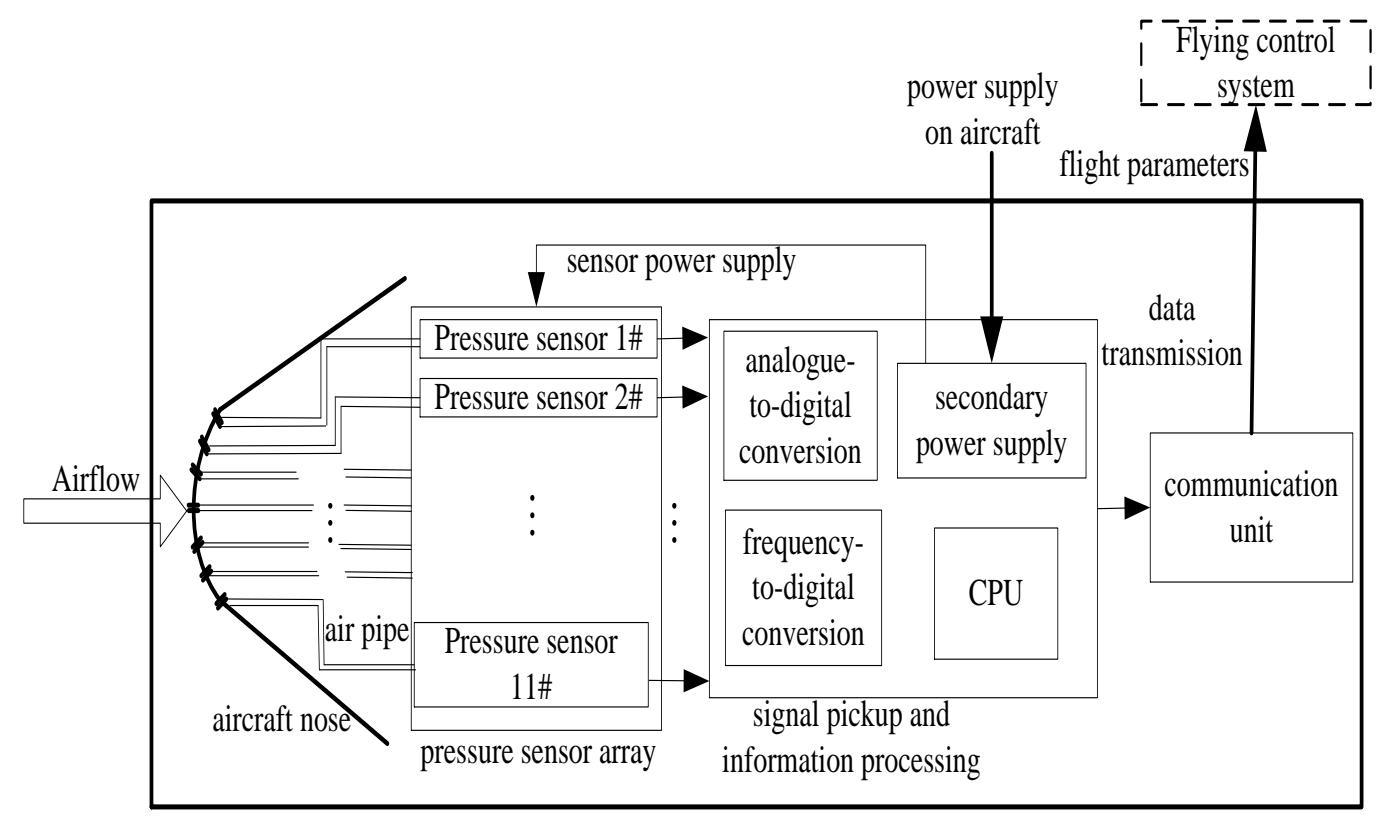

Figure 6. Electric and Airflow Connection Design of SVADS System

The prototype has been tested in the wind tunnel, in which the Mach number is from 0.1 to 1.0 , the angle of attack is from -15 degree to +15 degree, the angle of sideslip is also from -15 degree to +15 degree, and the altitude is from $0 \mathrm{~m}$ to $10000 \mathrm{~m}$. Above test is done under normal working situation, and enrich experimental data have been obtained. Actual faults of SVADS system is difficult to acquire, therefore, the faults data is produced by failure simulation on hardware platform. Based on the prototype of SVADS system, the emulation platform is built to study the detailed faults self-diagnosis algorithm. And the platform is based on PC and the functional chart is shown in Figure 7. 


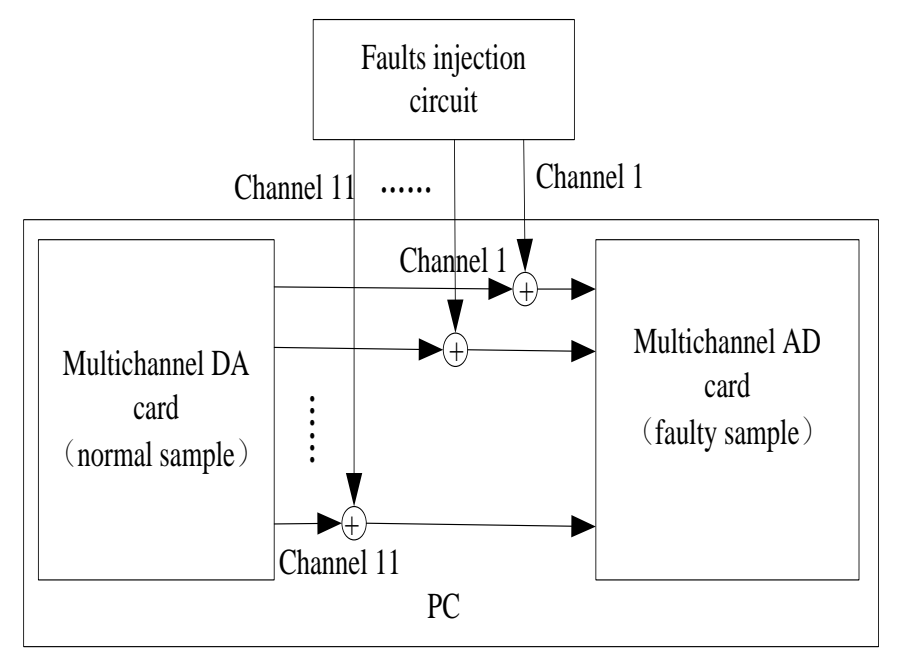

\section{Figure 7. Experimental Simulation Platforms for Studying Faults Self- Diagnosis Algorithm}

This simulation platform mainly includes the multichannel DA card which produce the normal pressure signal, faults injection circuit which is used to produce the faulty signal and add the faults into the normal one, multichannel AD card which can pick up the final faulty signals, PC which controls the faults injection way. The normal experiment test of prototype can be simulated by using the air data calibrator which can output multichannel gas pressure. Due to the limitation of our laboratory resource, this paper has done 9channel pressure experiment under different Mach number, different angle of attack, and angle of sideslip.

\subsection{Failure Mode Analysis of SVADS System}

The research of faults diagnosis algorithm needs faulty sample; however, real fault data is difficult to be captured or it takes some time to accumulate. The normal method is done by faults simulation based on above normal data sample, in which failure mode analysis is necessary. The main faults component includes the airflow pipes, pressure sensors, signal pickup and information processing unit, and 1553B based communication unit and their detailed faults information are follows:

1). The faults of airflow pipes often includes: the blockage of pressure ports caused by ice and dust will lead into the no change dynamic pressure when flying condition changes; pipe pressure leak caused by ageing of pipe material and seal ring will lead into sudden change of vertical speed and difficulty building signal reconstruction model; pipe pressure delay caused by the long distance will result in the hysteresis of actual pressure measurements values.

2).The faults of pressure sensors often includes: no output caused by inner broken wires and power failure will lead into the absence of frequency signals; large fluctuations caused by other residue or pollutants will result in larger fluctuations of frequency values; signal jump caused by lose effectiveness of vibration cylinder will lead into pressure jump; bias fault caused by the absence of temperature compensation will result in bias output.

3).The faults of signal pickup and information processing unit often includes: analogueto-digital conversion circuit fault caused by incorrect control command and wrong data bus will output the erroneous temperature information and further pressure measurements accuracy becomes worse; frequency-to-digital conversion circuit fault caused by the disorder of sequencing control will output wrong frequency and further pressure 
measurements values becomes wrong; CPU reset faults caused by weaker surge characteristic will no output in a short time; secondary power supply fault caused by the loss of power module will always lead into no output.

4). The faults of 1553B based communication unit faults include BC controller fault and RT terminal failure, which will not transmit the air data information to flying control system.

To sum up, the abnormal pressure outputs types mainly consists of larger fluctuation, jump, bias and constant output as shown in Figure 8.
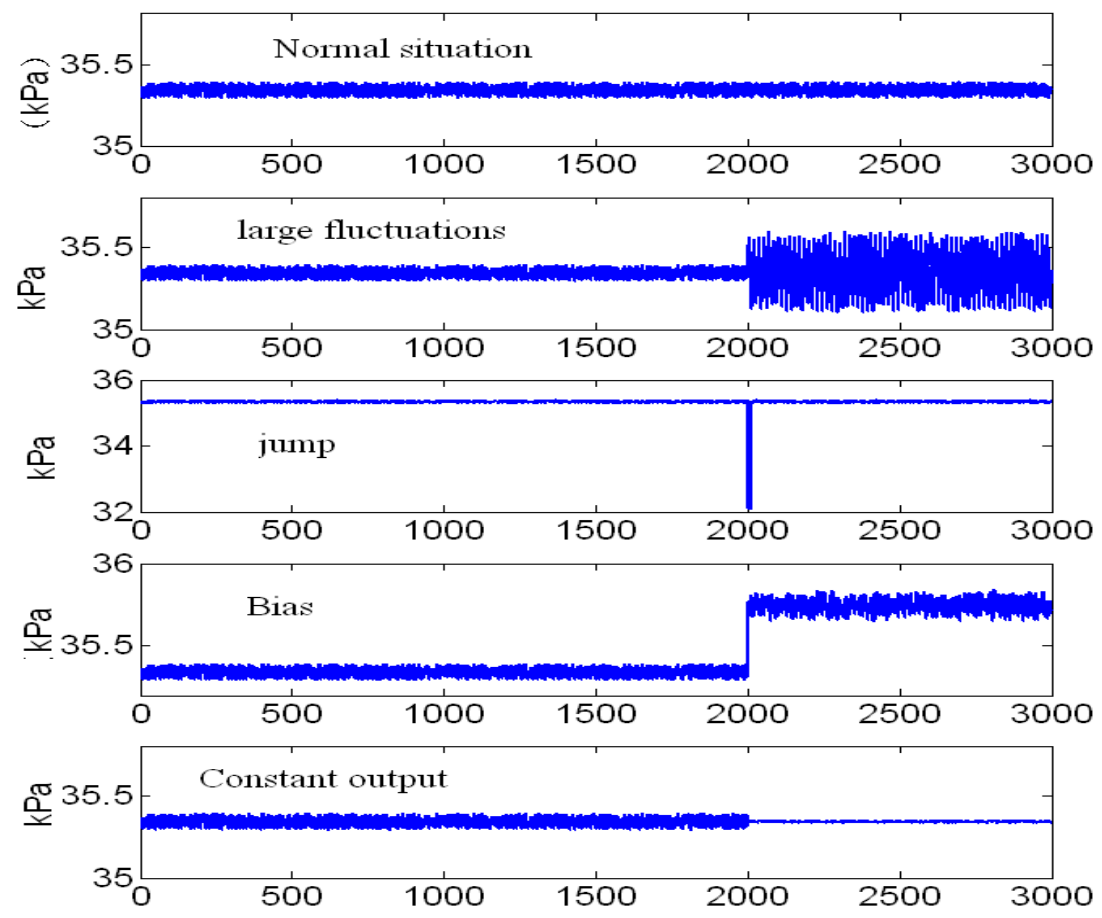

Figure 8. Common Types of Abnormal Pressure Outputs

\subsection{Faults Features Extraction Experiments and Results}

To verify the validity of EEMD based non-linear faults feature extraction, especially for the advantage of anti-mode mixing, both the simulation experiment and actual faults experiment are conducted.

The simulation experiment data is produced by synthetizing non-linear sine wave with different frequency and the performance of adaptive decomposing and high-frequency non-linear faults extraction will be test. The experiment process is follows: 1) data synthesis needs to be done by using equation (22) and (23), and the high-frequency sine signal is simulated to period noise disturbing fault wherein $t \in[1,2000]$ is the input variable, and $x_{1}, x_{2}$ are the output variable, and the composite function is expressed by $x=x_{1}+x_{2}$ as shown in Figure. 9; 2) to illustrate the anti-mode mixing, the signal decomposing performance of both EEMD and traditional EMD is compared and the results are shown in Figure. 10 and Figure.11.

$$
x_{1}=\sin \left(\frac{\pi}{50} t\right) \quad t \in[1,2000]
$$




$$
x_{2}= \begin{cases}\frac{1}{5} \sin \left(\frac{\pi}{5} t\right) & t \in[300,350] \\ \frac{1}{5} \sin \left(\frac{\pi}{5} t\right) & t \in[1700,1750] \\ 0 & \text { other }\end{cases}
$$
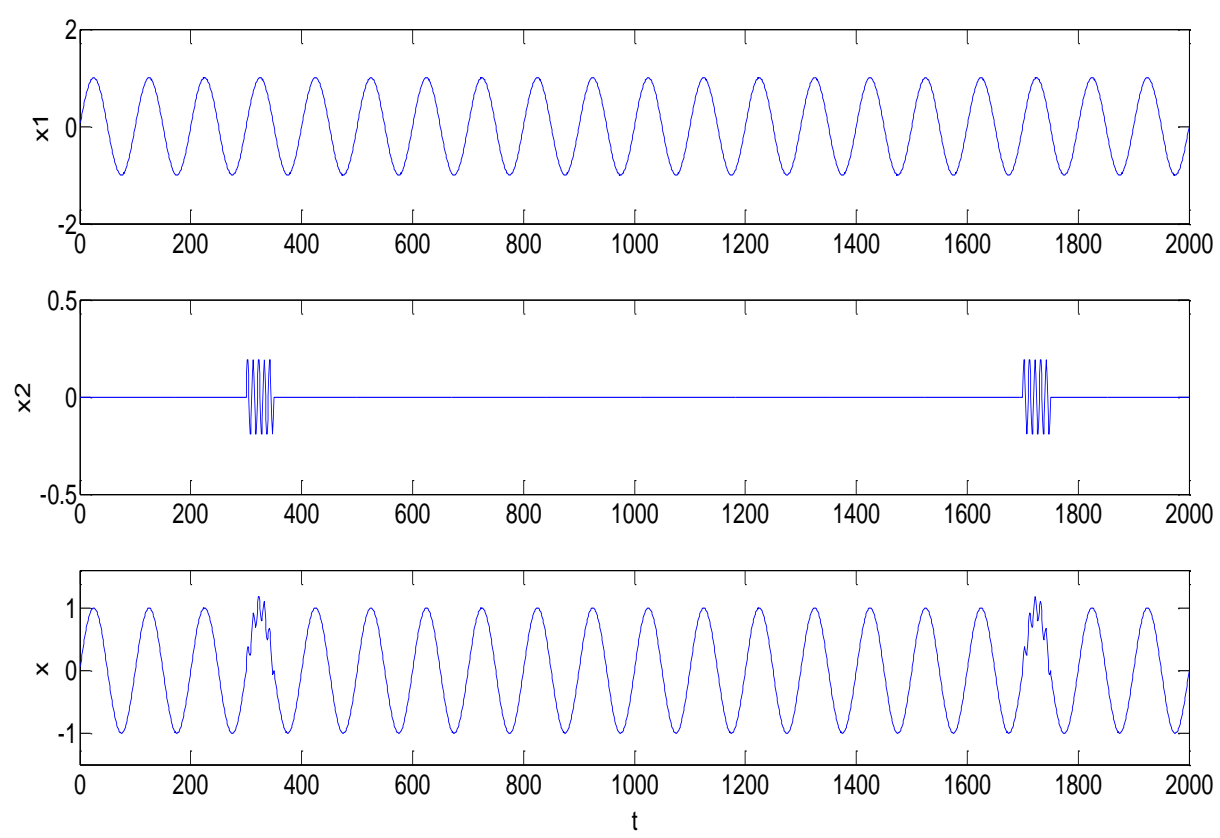

Figure 9. Simulated Faults Data Based on Standard Sine Functions

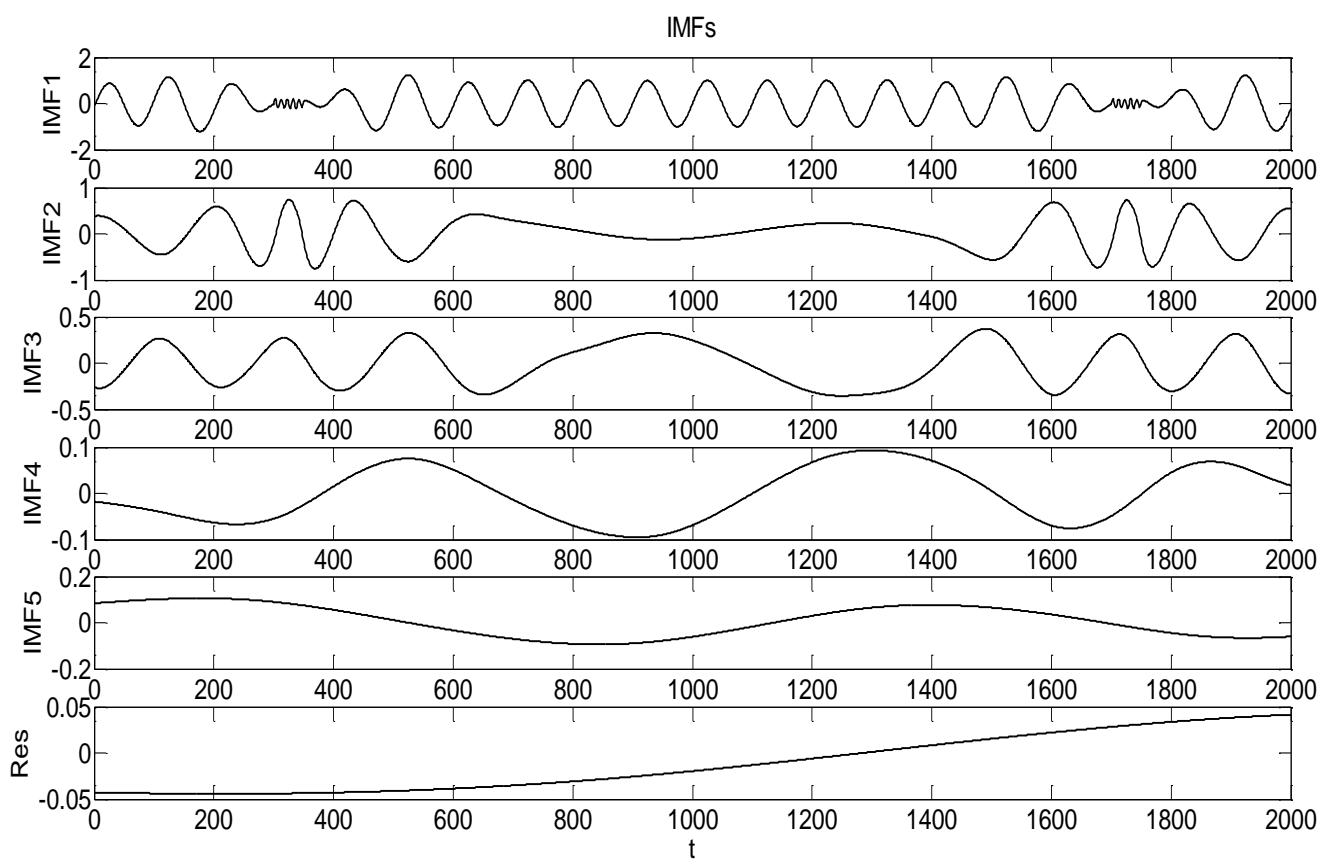

Figure 10. Adaptive Decomposing Results by Using Traditional EMD Method 


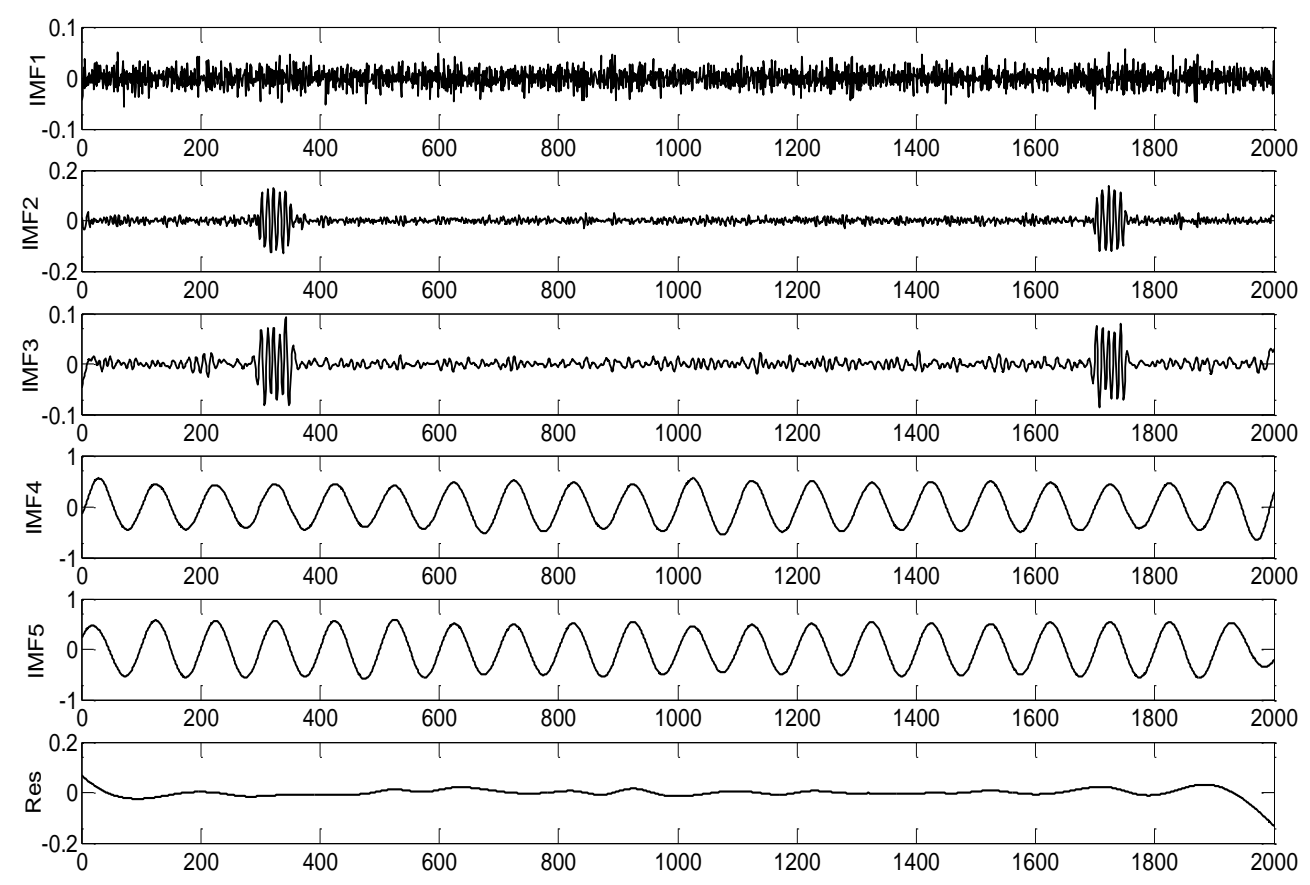

Figure 11. Adaptive Decomposing Results by Using Improved EEMD Method

From Figure 10, the decomposing results of the traditional EMD method produce the demonstrable deformation, and the original sine signal and small-amplitude sine which is used to simulate the periodic impact fault are decomposed into the same IMF1. The single IMF which includes multiple frequency components is exactly the mode mixing, which is very harmful to the non-linear faults feature extraction. Compared with the traditional EMD, the improved EEMD can restrain the above mode mixing effectively as shown in Figure 11, and the components with different frequency are decomposed into exactly distinct IMFs. The reason is that the frequency uniform distribution property of the added white noise in each trial of decompositions is fully employed in the EEMD method, and each individual trial will certainly change the number of extreme points of signal to avoid the mode mixing situation. The simulation experiment shows that EEMD can extract the energy feature of faults on different frequency component, and it is able to solve the problem of mode mixing and achieves an improved decomposition with physical meaning.

The actual pressure signals are then processed by using improved EEMD to pick up the feature vectors. The experiment process is: Firstly, under the condition that the flight altitude is $10000 \mathrm{~m}$, the angle of attack and sideslip are both 0 degree, and the Mach number is 0.8 , the fault-free pressure measurements of all channels are collected by using the multiple-channel air data system and our designed prototype of SVADS system. The mean value of the collected sample data is shown in Table 1. Secondly, taken the pressure channel 1 as an example, the faults are simulated by using our designed faults injection circuit in which the large pressure fluctuation, pressure jump, pressure bias and constant pressure output are produced. Thirdly, the abnormal signal with different fault types are collected by using our designed simulation platform shown in Figure 7, and the signal decompositions is conducted by the proposed EEMD; Fourthly, to further make the extracted features more obvious, the signal enhance work is done for the pressure bias fault, and their results are shown in Figure. 12. Lastly, acquire the energy and cutting ratio features on different frequency of above faulty signals and normal signal by using proposed method shown in Figure 13, and further take them as the foundation of following faults classification. 
Table 1. Mean Value of Fault-Free Pressure Sample Data

\begin{tabular}{|c|c|c|c|c|c|}
\hline Pressure channel & P1 & P2 & P3 & P4 & P5 \\
\hline Measurements $(\mathrm{kPa})$ & 35.342 & 38.707 & 40.309 & 38.707 & 35.341 \\
\hline Pressure channel & P6 & P7 & P8 & P9 & - \\
\hline Measurements $(\mathrm{kPa})$ & 35.345 & 38.709 & 38.709 & 35.345 & - \\
\hline
\end{tabular}

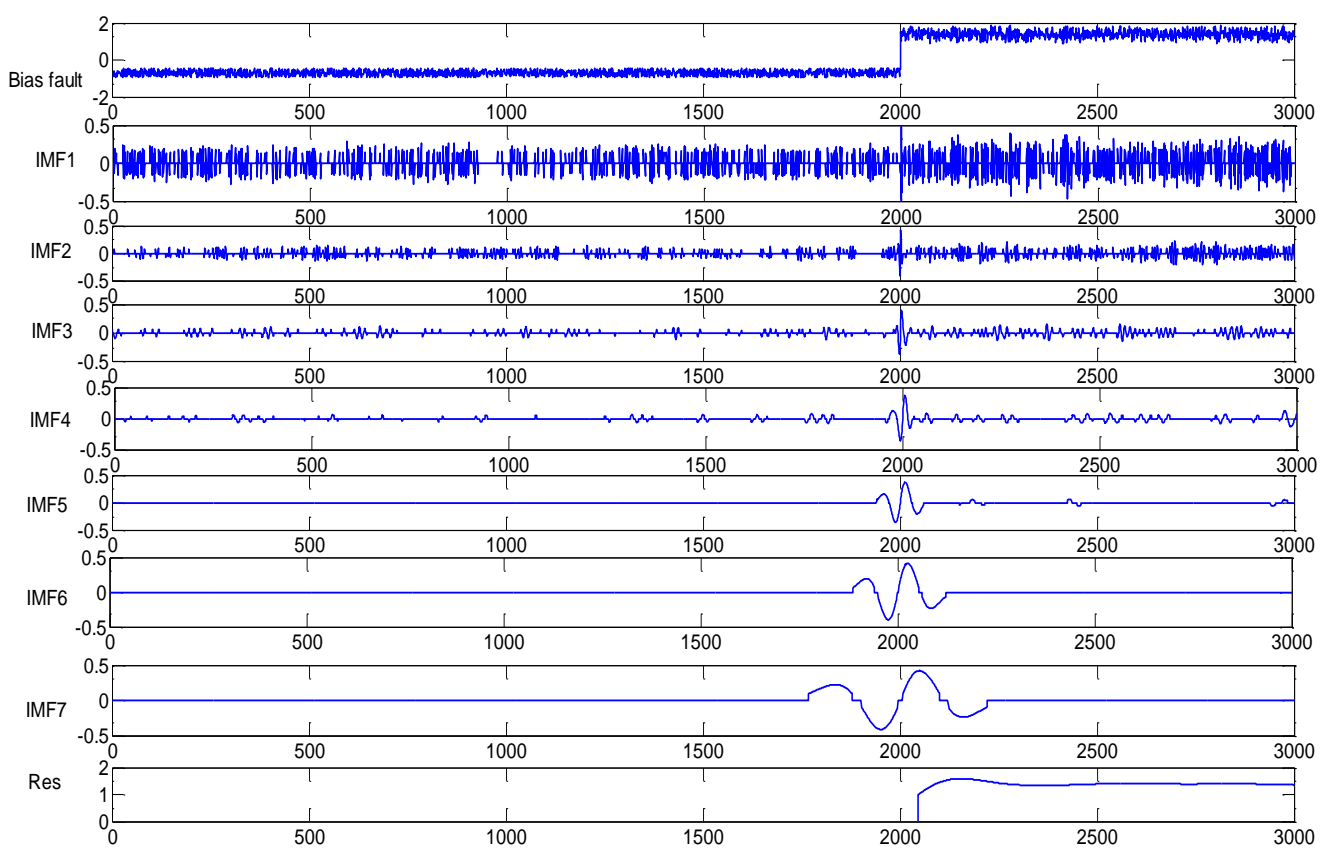

Figure 12. Decompositions and Enhancement of IMF Components of Pressure Bias Fault
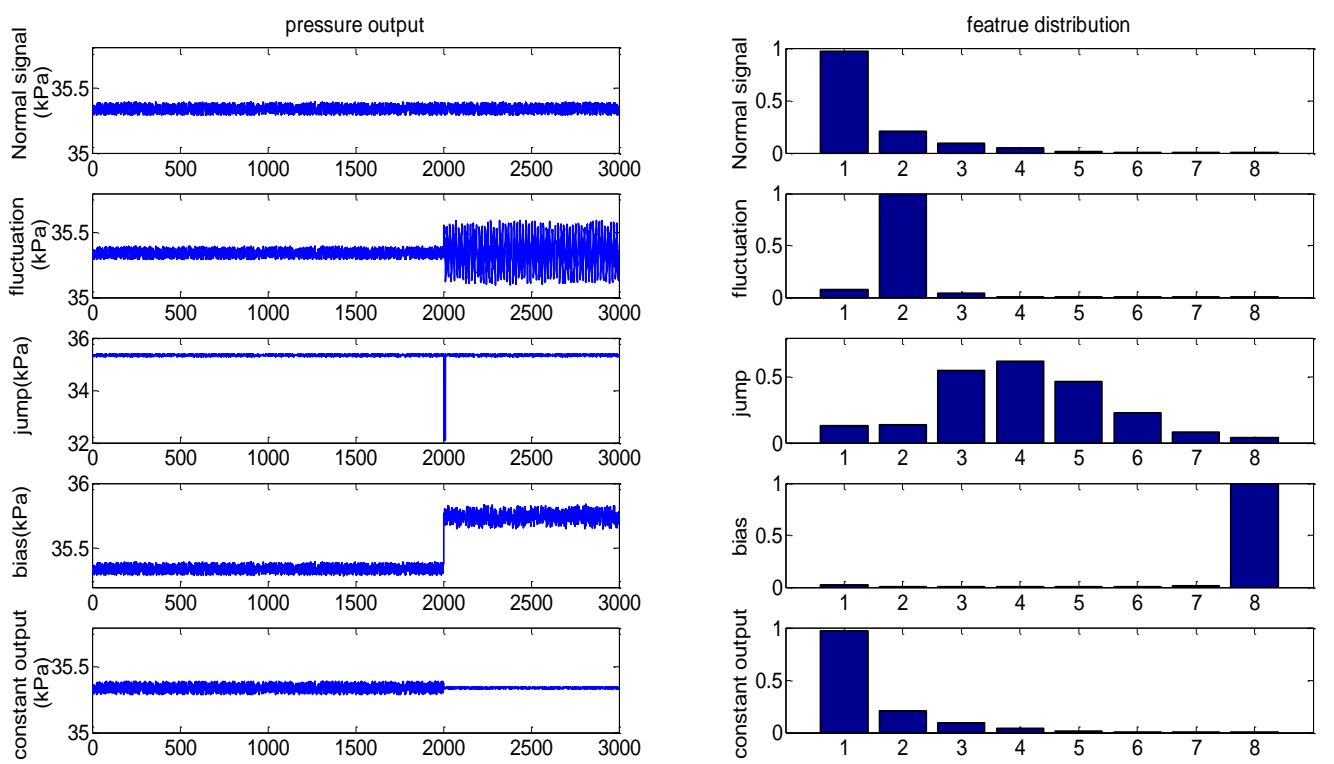

Figure 13. Different Pressure Faults Types and their Energy Features Based on the Proposed EEMD

In Figure 12, the IMFs component and the residual are obtained by using EEMD method, the bias fault feature is reflected on residual component, and through the further signal enhancement, the bias fault feature is more clearer, which benefits the energy 
extraction. Similarly, the energy feature of other faults types are obtained as shown in Figure 13. In Figure 13, different energy feature can correspond to different failure patterns, in which the more distinct the energy feature is, more helpful the patterns classify. However, the differences between normal signal and constant output are not quietly obvious. If other feature is not introduced, the wrong fault diagnosis results may occur. Aiming at this problem, the cutting ratio is taken as a supplement feature because pressure measurements are constant when pressure port is in blockage. To sum up, the working state of pressure channel and their feature vectors are generalized as shown in Table 2.

Table 2. Working State of Pressure Channel and their Feature Vectors

\begin{tabular}{|c|c|c|}
\hline Faults types & Pressure state & Feature vectors \\
\hline I & normal & 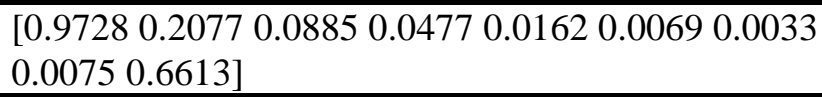 \\
\hline II & $\begin{array}{l}\text { large } \\
\text { fluctuation }\end{array}$ & $\begin{array}{l}{\left[\begin{array}{lllll}0.0744 & 0.9964 & 0.0392 & 0.0044 & 0.00160 .00070 .0006 \\
0.0023 & 0.6974\end{array}\right]}\end{array}$ \\
\hline III & jump & 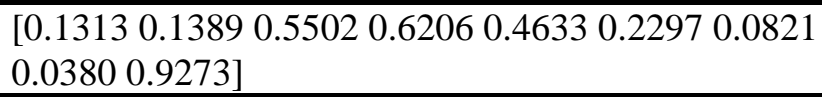 \\
\hline IV & bias & 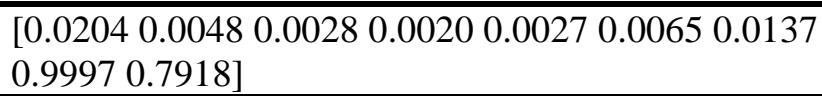 \\
\hline V & constant output & 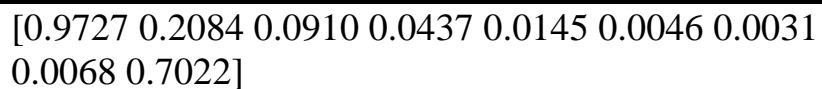 \\
\hline
\end{tabular}

\subsection{Faults Classification Experiments and Results}

To further verify the validity of MCRVM based multiple faults patterns classifier, especially for the uncertain diagnosis results expressed by probability, the faults diagnosis experiment are carried out.

The experiment process is follows: Firstly, under the normal situation that the flight altitude is $10000 \mathrm{~m}$, the angle of attack is from 0 degree to 10 degree, the angle of sideslip is 0 degree, and the Mach number is from 0.1 to 0.8 with the interval 0.1 , the fault-free pressure measurements of all channels are collected by using the multiple-channel air data system and our designed prototype of SVADS system, and the sum of each class of sample is 56 groups and the sample distribution is shown in Table 3. Secondly, taken the pressure channel 1 as an example, some fault data are simulated by using the faults injection circuit to produce the large pressure fluctuation, pressure jump, pressure bias and constant pressure output, in which the amplitude, starting time point, and ending time point of fault signal are all different to fully verify the performance of the proposed MCRVM method; Thirdly, based on the collected sample data, the optical kernel parameter of MCRVM model is selected by using 5-fold cross-validation way. Fourthly, taken the 140 groups of samples with odd number as the training data, the classifier based on MCRVM is built, and other 140 groups of sample with even number is regarded as the test sample, and the corresponding faults diagnosis results are shown in Table 4, the distribution of uncertain classification probability is shown in Figure 14. Lastly, to further verify the performance of small sample learning ability of the proposed MCRVM, the number of training sample decreased into 70, and the classification results are shown in Table 5 and their uncertain probability expression is shown in Figure 15. 
Table 3. Sample Distribution of Faults Diagnosis of SVADS System

\begin{tabular}{|c|c|c|c|c|c|c|}
\hline State & $\begin{array}{l}\text { sample } \\
\text { number }\end{array}$ & $\begin{array}{l}\text { training } \\
\text { sample } \\
\text { number } \\
\text { for cross- } \\
\text { validation }\end{array}$ & $\begin{array}{c}\text { test } \\
\text { sample } \\
\text { number } \\
\text { for cross- } \\
\text { validation }\end{array}$ & $\begin{array}{c}\text { training } \\
\text { sample } \\
\text { number } \\
\text { for small } \\
\text { sample } \\
\text { learning }\end{array}$ & $\begin{array}{c}\text { test } \\
\text { sample } \\
\text { number } \\
\text { for small } \\
\text { sample } \\
\text { learning }\end{array}$ & sample situation \\
\hline normal & 56 & 28 & 28 & 14 & 42 & \multirow{5}{*}{$\begin{array}{l}\text { angle of attack: } \\
0 、 2 、 3 、 5 、 7 、 \\
8 、 10 ; \text { angle of } \\
\text { sideslip: } 0 ; \text { Mach: } \\
0.1 \sim 0.8, \text { interval } \\
0.1 ; \\
\text { altitude: } 10000 \mathrm{~m} .\end{array}$} \\
\hline $\begin{array}{c}\text { large } \\
\text { fluctuation }\end{array}$ & 56 & 28 & 28 & 14 & 42 & \\
\hline jump & 56 & 28 & 28 & 14 & 42 & \\
\hline bias & 56 & 28 & 28 & 14 & 42 & \\
\hline $\begin{array}{c}\text { constant } \\
\text { output }\end{array}$ & 56 & 28 & 28 & 14 & 42 & \\
\hline
\end{tabular}

Table 4. Faults Diagnosis Results of MCRVM Model Under Larger Sample

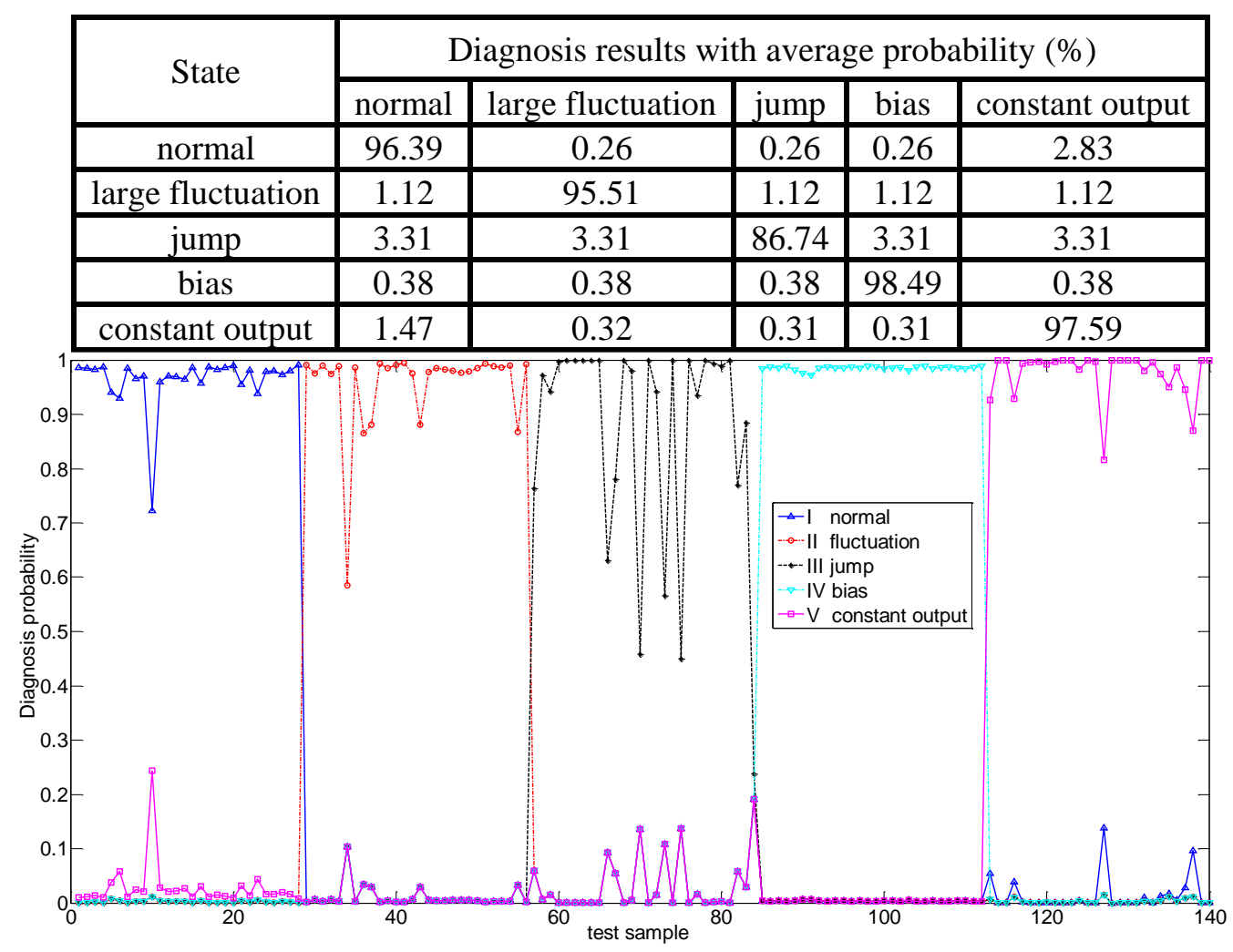

Figure 14. Faults Classification Results of MCRVM Model Under Larger
Sample

From Table 4 and Figure 14, the classification results of different faults types can be described by a probability form, which reveals the uncertainty of faults diagnosis results essentially. When the number of training sample is larger (about half of total sample number), the average accuracy that fault-free sample are identified correctly is about $96.39 \%$ while the probability that normal situation is identified as other real faults is $0.26 \%, 0.26 \%, 0.26 \%$, and $2.83 \%$ respectively. Similarly, the possibility that the pressure fluctuation, jump, bias, and constant output are diagnosed reasonably is all above $86 \%$. Therefore, the faults diagnosis result by using the proposed EEMD-MCRVM strategy is 
valid because the possibility of certain working sate which is diagnosed as their corresponding faults types is far larger than other types.

Table 5. Faults Diagnosis Results of MCRVM Model Under Small Sample

\begin{tabular}{|c|c|c|c|c|c|}
\hline \multirow{2}{*}{ State } & \multicolumn{5}{|c|}{ Diagnosis results with average probability (\%) } \\
\cline { 2 - 6 } & normal & large fluctuation & Jump & bias & constant output \\
\hline normal & 90.3 & 0.97 & 0.97 & 0.97 & 6.79 \\
\hline large fluctuation & 1.90 & 92.42 & 1.90 & 1.90 & 1.90 \\
\hline jump & 4.73 & 4.73 & 81.07 & 4.73 & 4.73 \\
\hline bias & 1.31 & 1.31 & 1.31 & 94.75 & 1.31 \\
\hline constant output & 6.67 & 1.85 & 1.85 & 1.85 & 87.77 \\
\hline
\end{tabular}

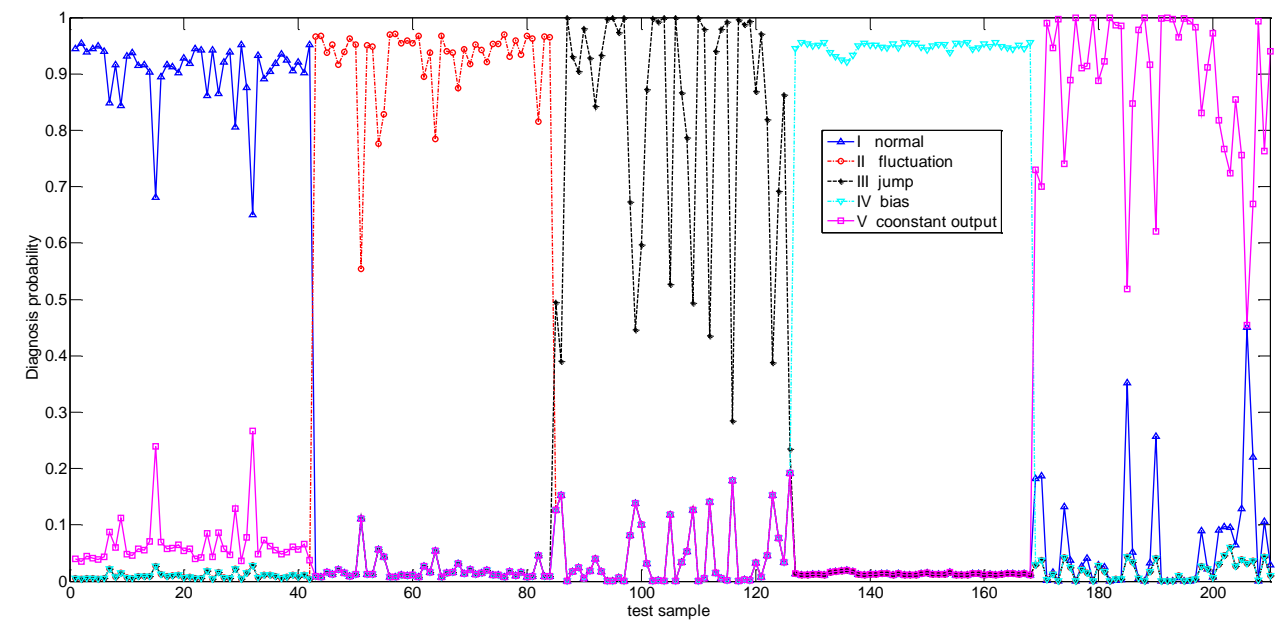

\section{Figure 15. Faults Classification Results of MCRVM Model Under Small Sample}

From Table 5 and Figure 15, when the number of training sample is smaller (about a quarter of total sample number), the average accuracy that fault-free sample is diagnosed as normal working condition is about $90.3 \%$. It is lower than larger sample situation; however, the possibility is still far larger than other faults types. Similarly, the diagnosis possibility of real faulty signals is also above $80 \%$. According to the principle that maximum possibility of special working state corresponds to the faults patterns exactly, the faults diagnosis results even under small sample situation are still proper by using the proposed EEMD-MCRVM strategy.

\section{Conclusions}

In this paper, a novel EEMD-MCRVM method has been presented to implement the faults diagnosis of self-validating air data sensing system. The advantages of the EEMDbased non-linear faults feature extraction and MCRVM-based multi-fault patterns classification under small samples are both combined in this strategy. The anti-mode mixing performance of EEMD is fully used to implement the adaptive decomposition and the energy feature of each sensitive IMF component is provided with clear physical meaning. By comparing with the traditional EMD method, the results demonstrate that the improved EEMD method is more effective to extract non-linear faults feature. The sparse characteristics and multiple patterns simultaneous classification of MCRVM with a novel uncertain expression way are properly employed for faults diagnosis of multiple faults types. By comparing with classification performance under both small sample and large 
sample situations, the results demonstrate that the proposed MCRVM method not only can describe the faults diagnosis results by using a probability form, but also it is provided with valid classification results completely even under small sample problem. Further, based on the detailed failure coverage analysis of the real SVADS system, the experimental platform is designed and some simulation experiments have been made to evaluate the faults diagnosis performance of the proposed strategy. The simulation experiment and real experiment are both completed, and different methods are compared and analyzed, the results demonstrate that the proposed EEMD-MCRVM methodology is more effective and more suitable to reveal the uncertainty of faults diagnosis problem essentially.

Based on the proposed EEMD-MCRVM methodology, the faults diagnosis by using the uncertain possibility form is implemented in novelty, and this uncertain diagnosis results can be further brought into the self-validating sensing system, which is of major significance for system reliability improvement and the following study on the dynamic or on-line uncertainty estimation and data recovery of SVADS system.

\section{Acknowledgments}

This work was supported by the National Natural Science Foundation of China (No.61401414).

\section{References}

[1] M. Jost, "Flush Air Data System-An Advanced Air Data System for the Aerospace Industry", 2004 AIAA Guidance, Navigation, and Control Conference and Exhibit, (2004).

[2] A. Srivastava, A. J. Meade and K. R. Long, "Learning Air Data Parameters for Flush Air Data Sensing Systems", Journal of Aerospace Computing Information \& Communication, vol. 9, no. 9, (2013), pp. 110-124.

[3] G. Qinghua, S. Zhengguang, D. Jingyu and Y. Jingchun, "Faults self-detection of Self-validating Flush Air Data Sensing System", International Journal of Control and Automation, (2015).

[4] S. Zhengguang, G. Qinghua, D. Jingyu and Y. Jingchun, "A Self-validating Flush Air Data Sensing System", 2015 12th International Conference on Fuzzy Systems and Knowledge Discovery (FSKD), Zhangjiajie, China, (2015).

[5] S. Zhengguang and W. Qi, "Status Self-validation of a Multifunctional Sensor Using a Multivariate Relevance Vector Machine and Predictive Filters", Measurement Science and Technology, vol. 24, no. 3, pp. 035103.

[6] S. Zhengguang and W. Qi, "A Novel Health Evaluation Strategy of Multifunctional Self-validating Sensors”, Sensors, vol. 13, no. 1, (2013), pp. 587-610.

[7] J. Madhavanpillai, R. Narayanan and H. C. Soudamini, "System and method for detecting and isolating faults in pressure sensing of flush air data system (FADS)", US Patent (No. 8930062B2), (2015).

[8] P. Freeman, P. Seiler and G. J. Balas, "Air data system fault modeling and detection", Control Engineering Practice, vol. 21, no. 10, (2013), pp. 1290-1301.

[9] J. Gligorijevic, D. Gajic and A. Brkovic, "Online Condition Monitoring of Bearings to Support Total Productive Maintenance in the Packaging Materials Industry", Sensors, vol. 16, no. 3, (2016), pp. 1-16.

[10] Y. P. Guo, Y. Xiong and G. C. Song, "Rolling Bearing Fault Diagnosis with EMD-Based Fault Characteristic Frequency Difference Analysis", Applied Mechanics \& Materials, vol. 596, (2014), pp. 437-441.

[11] B. Y. Vyas, B. Das and R. P. Maheshwari, "Improved Fault Classification in Series Compensated Transmission Line: Comparative Evaluation of Chebyshev Neural Network Training Algorithms", IEEE Transactions on Neural Networks \& Learning Systems, vol. 51, no. 3, (2014), pp. 379-385.

[12] Z. Liu, W. Guo and Z. Tang, "Multi-Sensor Data Fusion Using a Relevance Vector Machine Based on an Ant Colony for Gearbox Fault Detection", Sensors, vol. 15, no. 9, (2015), pp. 21857-75.

[13] Y. Lei and M. J. Zuo, "Fault diagnosis of rotating machinery using an improved HHT based on EEMD and sensitive IMFs", Measurement Science \& Technology, vol. 20, no. 12, (2009), pp. 314-317.

[14] Y. Cai, L. Jianhui, Z. Weihua and D. Jianming, "Faults Diagnostics of Railway Axle Bearings Based on IMF's Confidence Index Algorithm for Ensemble EMD", Sensors, vol. 15, no. 5, (2015), pp. 1099111011.

[15] P. Flandrin, G. Rilling and P. Goncalves, "Empirical mode decomposition as a filter bank", IEEE Signal Processing Letters, vol. 11, no. 2, (2004), pp. 112-114. 
[16] Z. Wu and N. E. Huang, "A study of the characteristics of white noise using the empirical mode decomposition method", Proceedings of the Royal Society A Mathematical Physical \& Engineering Sciences, vol. 460, no. 2046, (2004), pp. 1597-1611.

[17] M. E. Tipping, "The Relevance Vector Machine", Advances in Neural Information Processing System, vol. 12, no. 1, (2001), pp. 652-658.

[18] Y. Lei, Z. Liu and X. Wu, "Health condition identification of multi-stage planetary gearboxes using a mRVM-based method", Mechanical Systems \& Signal Processing, vol. 60, no. 1, (2015), pp. 289-300.

[19] I. Psorakis, T. Damoulas and M. A. Girolami, "Multiclass relevance vector machines: sparsity and accuracy", IEEE Transactions on Neural Networks, vol. 21, no. 10, (2010), pp. 1588-98. 
International Journal of Control and Automation Vol.10, No.9 (2017) 